\title{
THE DEGIORGI-NASH-MOSER TYPE OF ESTIMATE FOR PARABOLIC VOLTERRA INTEGRODIFFERENTIAL EQUATIONS
}

\author{
Bei Hu And Hong-Ming Yin
}

The DeGiorgi-Nash-Moser estimate plays a crucial role in the study of quasilinear elliptic and parabolic equations. In the present paper we shall show that this fundamental estimate holds for solutions of a linear parabolic Volterra integrodifferential equation:

$$
\frac{\partial u}{\partial t}=\frac{\partial}{\partial x_{i}}\left[a_{i j}(x, t) \frac{\partial u}{\partial x_{j}}\right]+\int_{0}^{t} \frac{\partial}{\partial x_{i}}\left[b_{i j}(x, t, \tau) \frac{\partial u}{\partial x_{j}}\right] d \tau,
$$

where $\left\{a_{i j}\right\}$ and $\left\{b_{i j}\right\}$ are only assumed to be measurable, bounded and $\left\{a_{i j}\right\}$ satisfy a strong ellipticity condition. The proof is based on $\mathcal{L}^{2, \mu}$ theory for parabolic equations. A global solvability result in the classical sense for a class of quasilinear parabolic integrodifferential equations is presented as an application of the general results.

\section{Introduction.}

In this paper we consider the following linear parabolic Volterra integrodifferential equation (PVIDE):

$$
\frac{\partial u}{\partial t}=\frac{\partial}{\partial x_{i}}\left[a_{i j}(x, t) \frac{\partial u}{\partial x_{j}}\right]+\int_{0}^{t} \frac{\partial}{\partial x_{i}}\left[b_{i j}(x, t, \tau) \frac{\partial u}{\partial x_{j}}\right] d \tau,(x, t) \in Q_{T}
$$

where $Q_{T}=\Omega \times(0, T], \Omega$ is a bounded domain in $R^{n}$ with boundary $S=$ $\partial \Omega \in C^{1}$.

The equation arises from a variety of mathematical models in engineering and physical sciences. For example, in the study of heat conduction in materials with memory, the classical Fourier's law is replaced by the following form (cf. [10]):

$$
\text { heat flux } \vec{q}=-d \nabla u-\int_{-\infty}^{t} \nabla[k(x, t, \tau) u(x, \tau)] d \tau,
$$

where $u$ is the temperature, $d$ the diffusion coefficient and the integral term represents the memory effect in the material. The conservation of energy 
implies that $u(x, t)$ will satisfy Eq. (1.1) with an inhomogeneous term, provided that the temperature is assumed to be known for $t \leq 0$. Another example is from a diffusion process in a glassy polymer (cf. [2], [7] and the references therein). Experiments indicate that the classical Fickian law does not match the diffusion process. A non-Fickian law (1.2) is used to model the problem, where $u(x, t)$ represents the concentration of the glassy polymer. Again the conservation of mass leads to a same type of equation as (1.1).

The study for this type of equations has drawn considerable attention (cf. $[1],[4]-[7],[16]-[17]$, etc.). The reader can find many more references in [10] and [13]. From a mathematical point of view, one would expect that the integral term should be dominated by the leading term in the Equation (1.1). Therefore, the theory of parabolic equations should be applicable to this type of equations. This is indeed true in some cases. Previous research strongly suggests that a solution of PVIDE has many similar properties to those of a parabolic equation. In [16] we have shown that the global Schauder estimate and a $W_{p}^{2,1}$-estimate hold. More recently, the author of [17] considered a general equation

$$
u_{t}=\operatorname{div} \vec{A}\left(x, t, u, u_{x}\right)+a\left(x, t, u, u_{x}\right)+\int_{0}^{t} \operatorname{div} \vec{B}\left(x, t, \tau, u, u_{x}\right) d \tau .
$$

Under some structure conditions on $\vec{A}, \vec{B}$ and $a$ similar to the case of parabolic equations, the existence of a unique weak solution is established. Regularity of the weak solution is also investigated. On the other hand, there are many essential differences between a PVIDE and a parabolic equation. For instance, the solution to a parabolic Volterra integrodifferential equation does not obey the maximum principle. A counterexample can be constructed without much difficulty. Therefore, it is a challenge to deduce an a priori $L^{\infty}$-bound of a solution. Unfortunately, it seems difficult to use the energy method to derive such a bound (see [17] for some special cases). Another essential difference concerns the regularity of solutions. By the DeGiorgiNash-Moser theory, we know that the solution of a parabolic equation with measurable coefficients $\left\{a_{i j}\right\}$ is Hölder continuous in the interior of the domain. No information is needed from initial and boundary data, as long as a weak solution exists. However, because of the nonlocal integral term in parabolic Volterra integrodifferential equations, the regularity of a solution strongly depends on the regularity of initial value.

The DeGiorgi-Nash-Moser's estimate is a milestone in the study of quasilinear elliptic and parabolic equations. In the present paper, we shall derive this fundamental a priori estimate for weak solutions of a PVIDE. The argument of the proof is based on various estimates in Campanato space 
$\mathcal{L}^{2, \mu}\left(Q_{T}\right)$, where $0<\mu<n+2$, which were developed recently for parabolic equations (cf. [18]). This $\mathcal{L}^{2, \mu}$ type estimate gives the precise dependency of Hölder norm of a solution, without a priori assuming $u(x, t)$ to be bounded. This theory enables us to use various integral estimates to replace the classical norm of a Hölder space. This method is widely used in the study of regularity of solutions of elliptic equations and systems (cf. [8], [11], [14], etc.). It turns out that this theory for parabolic equations is also very powerful.

This paper is prepared in the following manner. We will state the main results in Section 2 and present the proofs in Section 3. In Section 4, we employ the preceding results to obtain a global solvability result for a class of quasilinear PVIDE's in the classical sense.

\section{Notation and Main Results.}

We shall introduce some standard notation for reader's convenience:

A point $(x, t)$ in $Q_{T}$ will be denoted by $z$. The distance between two points $z_{1}=\left(x_{1}, t_{1}\right)$ and $z_{2}=\left(x_{2}, t_{2}\right)$ is equal to

$$
\max \left\{\left|x_{1}-x_{2}\right|,\left|t_{1}-t_{2}\right|^{\frac{1}{2}}\right\} \text {. }
$$

For $r>0$,

$$
\begin{aligned}
B_{r}\left(x_{0}\right) & =\left\{x \in R^{n}:\left|x-x_{0}\right|<r\right\} \\
\text { and } Q_{r}\left(z_{0}\right) & =B_{r}\left(x_{0}\right) \times\left[t_{0}-r^{2}, t_{0}\right], \quad \text { where } z_{0}=\left(x_{0}, t_{0}\right) .
\end{aligned}
$$

Let

$$
u_{z_{0}, r}=\frac{1}{\left|Q_{r}\left(z_{0}\right)\right|} \int_{Q_{r}\left(z_{0}\right)} u d z
$$

where $\left|Q_{r}\left(z_{0}\right)\right|$ denotes the Lesbegue measure of $Q_{r}\left(z_{0}\right)$ in $R^{n+1}$.

For $\mu>0$ and $z_{0}=\left(x_{0}, t_{0}\right)$, let

$$
[u]_{2, \mu, Q_{r}}=\left(\sup _{z_{0} \in Q_{r}, 0<\rho<r} \rho^{-\mu} \int_{Q_{\rho}\left(z_{0}\right)}\left|u-u_{z_{0}, \rho}\right|^{2} d z\right)^{\frac{1}{2}} .
$$

We shall use $Q_{r} \cap Q_{T}$ in the integration whenever $Q_{r}$ is not a subset of $Q_{T}$. The space $\mathcal{L}^{2, \mu}\left(Q_{r}\right)$ consists of all functions in $L^{2}\left(Q_{r}\right)$ such that

$$
[u]_{2, \mu, Q_{r}}<\infty
$$

$\mathcal{L}^{2, \mu}\left(Q_{r}\right)$ is a Banach space with the norm

$$
\|u\|_{2, \mu, Q_{r}}=\left\{\|u\|_{L^{2}\left(Q_{r}\right)}^{2}+[u]_{2, \mu, Q_{r}}^{2}\right\}^{\frac{1}{2}} .
$$


The Banach spaces $H^{1}(\Omega), L^{2}\left(0, T ; H^{1}(\Omega)\right), C^{\alpha, \frac{\alpha}{2}}\left(\bar{Q}_{T}\right)$, etc. are the same as those in [12]. Repeated subscript means a summation.

To the Equation (1.1), we append the following initial and boundary conditions:

$$
\begin{aligned}
& u(x, t)=g(x, t) \quad \text { on } \quad S_{T}=\partial \Omega \times[0, T], \\
& u(x, 0)=u_{0}(x) \quad \text { on } \Omega .
\end{aligned}
$$

Throughout this paper, the strong ellipticity condition is assumed regarding the measurable coefficients $a_{i j}$ :

$$
a_{i j}=a_{j i}, \quad \lambda|\xi|^{2} \leq a_{i j} \xi_{i} \xi_{j} \leq \Lambda|\xi|^{2} \quad \text { for } \xi \in R^{n},
$$

where $0<\lambda \leq \Lambda<\infty$ are constants.

Remark 1. Note that no ellipticity assumption is required regarding the coefficients $\left\{b_{i j}\right\}$.

Definition. A function $u \in L^{2}\left(0, T ; H^{1}(\Omega)\right)$ is said to be a weak solution of (1.1) in $Q_{T}$ satisfying (2.1)-(2.2), if $u-g \in L^{2}\left(0, T ; H_{0}^{1}(\Omega)\right)$ (here we assume that $g(x, t)$ can be extended to $\left.Q_{T}\right)$ and the following integral identity holds:

$$
\iint_{Q_{T}}\left[-u \varphi_{t}+\left[a_{i j} u_{x_{j}}+\int_{0}^{t} b_{i j} u_{x_{j}} d \tau\right] \varphi_{x_{i}}\right] d x d t=\int_{\Omega} u_{0} \varphi(x, 0) d x
$$

for any test function $\varphi \in C^{1}\left(\bar{Q}_{T}\right)$ with $\varphi(x, t)=0$ on $t=T$ and $S_{T}$.

The existence of a unique weak solution of (1.1), (2.1)-(2.2) can be proved by means of the finite element method (cf. [17]). Our objective is to obtain a priori estimates of the DeGiorgi-Nash-Moser type which imply the Hölder continuity of the weak solution $u(x, t)$ of (1.1), (2.1)-(2.2). We begin by obtaining an "interior estimate":

Theorem 2.1. Let $T>0$ and $Q \subset \subset Q_{T}$ with $d=\operatorname{dist}\left\{Q, S_{T}\right\}>0$. Let $a_{i j}(x, t), b_{i j}(x, t, \tau)$ be measurable and $\left\{a_{i j}\right\}$ satisfy (2.3). Assume that

$$
\sum_{i, j=1}^{n}\left[\left\|a_{i j}\right\|_{L^{\infty}\left(Q_{T}\right)}+\left\|b_{i j}\right\|_{L^{\infty}\left(Q_{T}\right)}\right] \leq M<\infty,
$$

and that

$$
\left\|\nabla u_{0}\right\|_{\mathcal{L}^{2,\left(\mu_{0}-2\right)+}(\Omega)}<\infty,
$$

where $\mu_{0}=n+2 \beta$ for some $\beta \in(0,1)$. Then there exist constants $\alpha \in(0,1)$ and $C>0$ such that any $L^{2}\left(0, T ; H^{1}(\Omega)\right)$ solution $u(x, t)$ of (1.1), (2.1)-(2.2) on $Q_{T}$ satisfies

$$
\|u\|_{C^{\alpha, \frac{\alpha}{2}(Q)}} \leq C
$$


where the constant $C$ and the Hölder exponent $\alpha$ depend only on $d, M, \lambda, T$, $\|u\|_{L^{2}\left(0, T ; H^{1}(\Omega)\right)}$ and $\left\|\nabla u_{0}\right\|_{\mathcal{L}^{2,\left(\mu_{0}-2\right)+}{ }_{(\Omega)}}$.

Remark 2. As we mentioned in Introduction, the interior estimate in Theorem 2.1 holds actually down to the bottom $t=0$, but away from the lateral boundary $S_{T}$. This interior estimate is not valid without the required regularity on $u_{0}$.

Next, we obtain a DeGiorgi-Nash-Moser type estimate valid up to the boundary:

Theorem 2.2. In addition to assumptions of Theorem 2.1, assume that there exists a function $\psi(x, t) \in H^{1}\left(0, T ; H^{1}(\Omega)\right)$ such that

$$
\psi(x, t)=g(x, t) \text { on } S_{T}, \text { and } \psi(x, 0)=u_{0}(x) \text { on } \Omega
$$

in the sense of trace. If $\psi_{t}, \psi_{x_{i}} \in \mathcal{L}^{2, \mu_{0}}\left(Q_{T}\right)$ for $i=1, \cdots, n$, then there exist constants $C$ and $\alpha \in(0,1)$ such that

$$
\|u\|_{C^{\alpha, \frac{\alpha}{2}}\left(\bar{Q}_{T}\right)} \leq C
$$

where the constants $C$ and $\alpha$ depend only on $\left\|\psi_{t}\right\|_{\mathcal{L}^{2, \mu_{0}}\left(Q_{T}\right)}+\|\nabla \psi\|_{\mathcal{L}^{2, \mu_{0}}\left(Q_{T}\right)}$ and the data in Theorem 2.1.

Remark 3. The estimate (2.4) is also true for the following conormal boundary condition:

$$
a_{i j} u_{x_{j}} \cos \left(n_{i}, x_{i}\right)=g(x, t) \text {, where } \vec{n} \text { is the outward normal on } S,
$$

provided that $g(x, t)$ is uniformly bounded.

Remark 4. The equation is linear for $u$. By renormalization of the equation, one can write the dependence on the initial data in the interior estimate as follows.

$$
\|u\|_{C^{\alpha, \frac{\alpha}{2}}} \leq C\left(\|u\|_{L^{2}\left(0, T ; H^{1}(\Omega)\right)}+\left\|\nabla u_{0}\right\|_{\mathcal{L}^{2, \mu_{0}-2}(\Omega)}\right) .
$$

A similar remark also applies to the global estimate.

Remark 5. We stated our Theorems 2.1 and 2.2 for the operator containing only the highest order derivative. All the results can be extended without any difficulty to the following operator

$$
\begin{aligned}
& L[u] \\
& =\frac{\partial u}{\partial t}-\left\{\frac{\partial}{\partial x_{i}}\left[a_{i j}(x, t) \frac{\partial u}{\partial x_{j}}\right]+b_{i}(x, t) \frac{\partial u}{\partial x_{i}}+c u\right.
\end{aligned}
$$




$$
\begin{aligned}
& \left.+\int_{0}^{t}\left(\frac{\partial}{\partial x_{i}}\left[b_{i j}(x, t, \tau) \frac{\partial u}{\partial x_{j}}\right]+d_{i}(x, t, \tau) \frac{\partial u}{\partial x_{i}}(x, \tau)+e(x, t, \tau) u(x, \tau)\right) d \tau\right\} \\
& +\sum_{j} \frac{\partial f_{j}}{\partial x_{j}}+f
\end{aligned}
$$

provided that suitable conditions on the coefficients of lower order terms hold.

\section{Proofs.}

We shall first state the following interior DeGiorgi-Nash-Moser type estimate. Let $x_{0} \in \Omega$ be arbitrary with $d=\operatorname{dist}\left\{x_{0}, \partial \Omega\right\}>0$. Let $Q_{R, \widetilde{T}}=$ $B_{R}\left(x_{0}\right) \times\left(\widetilde{T}-R^{2}, \widetilde{T}\right]$, where $\widetilde{T} \in[0, T]$. We shall restrict $R \leq \frac{d}{2}$.

Lemma 3.1. Let $v(x, t)$ be a solution to the following equation (no initialboundary conditions needed)

$$
v_{t}=\frac{\partial}{\partial x_{j}}\left[a_{i j}(x, t) \frac{\partial v}{\partial x_{i}}\right] \quad \text { in } Q_{R, \widetilde{T}},
$$

then there exists constants $C_{1}>0$ and $\alpha \in(0,1)$ such that for any $r \in(0, R]$

$$
\iint_{Q_{r, \widetilde{T}}}|\nabla v|^{2} d x d t \leq C_{1}\left(\frac{r}{R}\right)^{n+2 \alpha} \iint_{Q_{R, \widetilde{T}}}|\nabla v|^{2} d x d t
$$

where the constants $C_{1}$ and $\alpha$ depend only on $\lambda$ and $\Lambda$, they are independent of $r$ and $R$.

This is the same as Lemma 2.4 in [18]. We shall not repeat the proof.

For the convenience of the proof we extend the solution $u(x, t)$ to $t<0$ by letting

$$
u(x, t) \equiv u_{0}(x) \text { for } t<0 .
$$

We also define

$$
a_{i j}(x, t) \equiv \lambda \delta_{i j} \quad \text { for } t<0 .
$$

Our key estimate is the next lemma. As indicated in the introduction, the estimates will have to depend on the initial data, owing to the nonlocal nature of the equation.

Lemma 3.2. Let $u(x, t) \in L^{2}\left(0, T ; H^{1}(\Omega)\right)$ be a weak solution of Equation (1.1). Suppose that $u_{0} \in H^{1}(\Omega)$ such that for any $x_{0} \in \Omega$

$$
\int_{B_{R}\left(x_{0}\right)}\left|\nabla u_{0}\right|^{2} d x \leq M_{1} R^{n-2+2 \beta} \quad \text { for any } 0<R<\frac{d}{2},
$$


(without loss of generality, we may assume that $\beta<\alpha$, where $\alpha$ is the constant in Lemma 3.1).

(1). (Interior estimates) If dist $\left\{Q_{2 R, \widetilde{T}}, S_{T}\right\}>0$, then

$$
\iint_{Q_{R, \widetilde{T}}}|\nabla u|^{2} d x d t \leq C R^{n+2 \beta}
$$

where the constant $C$ depends only on $\lambda, \Lambda, d$, the constant $M$ in Theorem 2.1, the constant $M_{1}$, and the $L^{2}\left(0, T ; H^{1}(\Omega)\right)$-norm of the solution $u$.

(2). (Boundary estimates) Moreover, if u satisfies the boundary condition as specified in Theorem 2.2, then for any $\widetilde{T}>0$,

$$
\iint_{Q_{R, \widetilde{T}} \cap(\Omega \times[-1, \widetilde{T}])}|\nabla u|^{2} d x d t \leq C R^{n+2 \beta},
$$

where the constant $C$ depends only on the boundary data in addition to the quantities in the interior estimate (1) above.

Proof. Let $v(x, t)$ be the solution of the following problem:

$$
\begin{aligned}
v_{t} & =\frac{\partial}{\partial x_{j}}\left[a_{i j}(x, t) \frac{\partial v}{\partial x_{i}}\right] & & \text { in } Q_{R, \widetilde{T}}, \\
v(x, t) & =u(x, t) & & \text { on } \partial_{p} Q_{R, \widetilde{T}} .
\end{aligned}
$$

It is then clear that $u-v$ satisfies the following equation in the weak sense

$$
(u-v)_{t}=\frac{\partial}{\partial x_{j}}\left[a_{i j} \frac{\partial(u-v)}{\partial x_{i}}\right]+\frac{\partial}{\partial x_{j}} b_{j}(x, t)+\frac{\partial}{\partial x_{j}} f_{j}(x, t),
$$

where

$$
\begin{aligned}
& b_{j}(x, t)= \begin{cases}\int_{0}^{t} b_{i j}(x, t, \tau) \frac{\partial u(x, \tau)}{\partial x_{i}} d \tau & \text { for } t \geq 0 \\
0 & \text { for } t<0,\end{cases} \\
& f_{j}(x, t)= \begin{cases}0 & \text { for } t \geq 0 \\
-\lambda \frac{\partial u_{0}}{\partial x_{j}}(x) & \text { for } t<0 .\end{cases}
\end{aligned}
$$

Multiplying this equation with $u-v$ and integrating over $Q_{R, \widetilde{T}}$, we obtain

$$
\begin{aligned}
& \sup _{\widetilde{T}-R^{2} \leq \tau \leq \widetilde{T}} \int_{B_{R}}(u-v)^{2} d x+\frac{\lambda}{2} \iint_{Q_{R, \widetilde{T}}}|\nabla(u-v)|^{2} d x d t \\
& \leq \frac{1}{2 \lambda} \sum_{j} \iint_{Q_{R, \widetilde{T}}}\left(b_{j}+f_{j}\right)^{2} d x d t .
\end{aligned}
$$


Observe that the estimate in Lemma 3.1 holds for $v$ independently of the initial and boundary conditions on the parabolic boundary $\partial_{p} Q_{R, \widetilde{T}}$. Indeed, for any $r \leq \frac{R}{2}$ we have from Lemma 3.1

$$
\iint_{Q_{r, \widetilde{T}}}|\nabla v|^{2} d x d t \leq C_{1}\left(\frac{r}{R}\right)^{n+2 \alpha} \iint_{Q_{R, \widetilde{T}}}|\nabla v|^{2} d x d t
$$

When $\frac{R}{2} \leq r \leq R$, the estimate is trivial. Using Lemma 3.1 and (3.3), we now get, for any $r \leq R$,

$$
\begin{aligned}
& \iint_{Q_{r, \widetilde{T}}}|\nabla u|^{2} d x d t \\
& \leq 2 \iint_{Q_{r, \widetilde{T}}}|\nabla v|^{2} d x d t+2 \iint_{Q_{r, \widetilde{T}}}|\nabla(u-v)|^{2} d x d t \\
& \leq 2 C_{1}\left(\frac{r}{R}\right)^{n+2 \alpha} \iint_{Q_{R, \widetilde{T}}}|\nabla v|^{2} d x d t+2 \iint_{Q_{R, \widetilde{T}}}|\nabla(u-v)|^{2} d x d t \\
& (3.4) \\
& \leq 4 C_{1}\left(\frac{r}{R}\right)^{n+2 \alpha} \iint_{Q_{R, \widetilde{T}}}|\nabla u|^{2} d x d t \\
& \quad+\left[4 C_{1}\left(\frac{r}{R}\right)^{n+2 \alpha}+2\right] \iint_{Q_{R, \widetilde{T}}}|\nabla(u-v)|^{2} d x d t \\
& \leq 4 C_{1}\left(\frac{r}{R}\right)^{n+2 \alpha} \iint_{Q_{R, \widetilde{T}}}|\nabla u|^{2} d x d t+\left(4 C_{1}+2\right) \iint_{Q_{R, \widetilde{T}}}|\nabla(u-v)|^{2} d x d t \\
& \leq 4 C_{1}\left(\frac{r}{R}\right)^{n+2 \alpha} \iint_{Q_{R, \widetilde{T}}}|\nabla u|^{2} d x d t+\frac{2\left(2 C_{1}+1\right)}{\lambda^{2}} \sum_{j} \iint_{Q_{R, \widetilde{T}}}\left|b_{j}+f_{j}\right|^{2} d x d t .
\end{aligned}
$$

We now estimate $b_{j}(x, t)$. Recalling that $M$ is the bound for the coefficients $b_{i j}(x, t)$, we have (by Hölder's inequality)

$$
\left|b_{j}(x, t)\right|^{2} \leq M^{2} \widetilde{T} \int_{0}^{t}\left|\frac{\partial u}{\partial x_{i}}\right|^{2}(x, \tau) d \tau \leq M^{2} \widetilde{T} \int_{0}^{\widetilde{T}}\left|\frac{\partial u}{\partial x_{i}}\right|^{2}(x, \tau) d \tau
$$

It follows that

$$
\begin{aligned}
& \iint_{Q_{R, \widetilde{T}}}\left|b_{j}(x, t)\right|^{2} d x d t \\
& \leq M^{2} \widetilde{T} R^{2} \int_{0}^{\widetilde{T}} \int_{B_{R}}|\nabla u|^{2} d x d t
\end{aligned}
$$




$$
\begin{aligned}
& \leq M^{2} \widetilde{T} R^{2} \sum_{k=0}^{\left[\widetilde{T} / R^{2}\right]} \int_{\widetilde{T}-(k+1) R^{2}}^{\widetilde{T}-k R^{2}} \int_{B_{R}}|\nabla u|^{2} d x d t \\
& \leq M^{2} \widetilde{T} R^{2}\left(\left[\widetilde{T} / R^{2}\right]+1\right) \max _{0 \leq k \leq\left[\widetilde{T} / R^{2}\right]} \int_{\widetilde{T}-(k+1) R^{2}}^{\widetilde{T}-k R^{2}} \int_{B_{R}}|\nabla u|^{2} d x d t \\
& \leq M^{2} \widetilde{T}\left(\widetilde{T}+R^{2}\right) \max _{0 \leq k \leq\left[\widetilde{T} / R^{2}\right]} \int_{\widetilde{T}-(k+1) R^{2}}^{T} \int_{B_{R}}|\nabla u|^{2} d x d t,
\end{aligned}
$$

where $\left[\widetilde{T} / R^{2}\right]$ is the integer part of $\widetilde{T} / R^{2}$.

By assumption, we have

$$
\iint_{Q_{R, \widetilde{T}}}\left|f_{j}(x, t)\right|^{2} d x d t \leq C_{2} R^{n+2 \beta} .
$$

Now let $T^{*}>0$ to be specified later and $x_{0} \in \Omega$ such that $B_{R}\left(x_{0}\right) \subset \Omega$. Set (notice that $u(x, t)$ has already been extended to $t<0$ )

$$
g\left(R, x_{0}\right)=\sup _{0 \leq t \leq T^{*}} \int_{t-R^{2}}^{t} \int_{B_{R}\left(x_{0}\right)}|\nabla u|^{2} d x d t .
$$

Then by (3.4), (3.6) and (3.7),

$$
\begin{aligned}
g\left(r, x_{0}\right) \leq & {\left[4 C_{1}\left(\frac{r}{R}\right)^{n+2 \alpha}+\frac{4\left(2 C_{1}+1\right)}{\lambda^{2}} M^{2} T^{*}\left(T^{*}+R^{2}\right)\right] g\left(R, x_{0}\right) } \\
& +C^{*} R^{n+2 \beta}
\end{aligned}
$$

Note that we have assumed that $\beta<\alpha$. By [8] (Lemma 2.1, p. 86), there exists $\varepsilon_{0}>0$, depending only on $\alpha, \beta$ and $C_{1}\left(\varepsilon_{0}\right.$ is independent of $\left.C^{*}\right)$, such that if

$$
\frac{\left(4 C_{1}+2\right)}{\lambda^{2}} M^{2} T^{*}\left(T^{*}+R^{2}\right) \leq \varepsilon_{0}
$$

then

$$
g\left(r, x_{0}\right) \leq C\left[\left(\frac{r}{R}\right)^{n+2 \beta} g\left(R, x_{0}\right)+C^{*} r^{n+2 \beta}\right],
$$

where the constant $C$ depends only on $\alpha, \beta$ and $C_{1}$. The condition (3.10) is satisfied if we choose $T^{*}$ to be suitably small. By a well-known iteration process (cf. [8]), we have

$$
\iint_{Q_{R, \widetilde{T}}}|\nabla u|^{2} d x d t \leq C R^{n+2 \beta} \quad \text { if } 0 \leq \widetilde{T} \leq T^{*}, B_{R} \subset \Omega,
$$


where $C$ depends only on $L^{2}\left(0, T ; H^{1}(\Omega)\right)$-norm of $u(x, t), \beta$ and the constant in (3.11).

Notice that the estimate (3.12) is good for small $T^{*}$ satisfying (3.10). Next we want to show that this estimate can be extended step-by-step in $t$ direction with exactly the same step length (equal to $T^{*}$ ) in each step. Therefore the estimate is valid for any given finite time interval.

Suppose that the estimate $(3.12)$ is correct for $0<\widetilde{T} \leq k T^{*}$. We rewrite the equation as

$$
u_{t}=\frac{\partial}{\partial x_{j}}\left[a_{i j}(x, t) \frac{\partial u}{\partial x_{i}}\right]+\left(\int_{k T^{*}}^{t}+\int_{0}^{k T^{*}}\right) \frac{\partial}{\partial x_{i}}\left[b_{i j}(x, t, \tau) \frac{\partial u}{\partial x_{j}}\right] d \tau .
$$

All the previous estimates are still valid, with

$$
f_{j}(x, t)=\sum_{i} \int_{0}^{k T^{*}} b_{i j}(x, t, \tau) \frac{\partial u(x, \tau)}{\partial x_{i}} d \tau .
$$

By the induction hypothesis we have, for $k T^{*}<\widetilde{T}<(k+1) T^{*}$,

$$
\begin{aligned}
\sum_{j} \iint_{Q_{R, \widetilde{T}}}\left|f_{j}(x, t)\right|^{2} d x d t & \leq M^{2} R^{2} k T^{*} \int_{0}^{k T^{*}} \int_{B_{R}}|\nabla u|^{2} d x d \tau \\
& \leq M^{2} R^{2} k T^{*}\left(\left[\frac{k T^{*}}{R^{2}}\right]+1\right) R^{n+2 \beta} \\
& \leq C(k) R^{n+2 \beta}
\end{aligned}
$$

where the constant $C(k)$ may increase with each step. Now the exact procedure as in (3.1) to (3.10) implies that

$$
g\left(r, x_{0}\right) \leq\left[4 C_{1}\left(\frac{r}{R}\right)^{n+2 \alpha}+\varepsilon_{0}\right] g\left(R, x_{0}\right)+C \cdot C(k) R^{n+2 \beta} .
$$

Since the choice of $\varepsilon_{0}$ in (3.10) is independent of the constant $C \cdot C(k)$, the estimate (3.12) extends to $k T^{*}<\widetilde{T}<(k+1) T^{*}$. This proves the interior estimates.

From the proof, we see that the estimates (3.12) extends to the lateral boundary if Lemma 3.1 extends to the lateral boundary. We know that such results are valid (see [11]) if either a Dirichlet or Neumann boundary condition is imposed on the lateral boundary and the boundary value satisfies the condition stated in Theorem 2.2. We shall skip the detail here.

Finally, let us finish the Proof of Theorems 2.1 and 2.2. In order to show that $u(x, t)$ is Hölder continuous, we shall employ the parabolic embedding 
theorem (Lemma 2.6 in [18]). By applying Lemma 2.6 in [18], we have for any $Q_{\rho} \subset Q_{T}$ with $\operatorname{dist}\left\{B_{2 \rho}, \partial \Omega\right\}>0$,

$$
\begin{aligned}
& \iint_{Q_{\rho}}\left(u-u_{z_{0}, \rho}\right)^{2} d z \\
& \leq C \rho^{2} \iint_{Q_{2 \rho}}|\nabla u|^{2} d z+C \rho^{2} \iint_{Q_{2 \rho}} \sum_{j=1}^{n}\left[f_{j}^{2}+b_{j}^{2}\right] d z \\
& \leq C \rho^{2}\left[\iint_{Q_{2 \rho}}|\nabla u|^{2} d z+\rho^{2} \int_{B_{2 \rho}} \int_{0}^{t_{0}}|\nabla u|^{2} d \tau d z+\rho^{2} \int_{B_{2 \rho}}\left|\nabla u_{0}\right|^{2} d x\right] \\
& \leq C \rho^{n+2+2 \beta},
\end{aligned}
$$

where at the final step we have used the estimates for $f_{j}$ and $b_{j}$.

As $\mathcal{L}^{2, n+2+2 \beta}(Q)$ for any $\beta \in(0,1)$ is equivalent to $C^{\beta, \frac{\beta}{2}}(\bar{Q})$ (cf. Proposition $\mathrm{A}$ in [14]) it follows that $u(x, t)$ is Hölder continuous in $Q_{r}=B_{r}\left(x_{0}\right) \times$ $[0, T]$. As $r$ is independent of solution and $x_{0}$ is arbitrary in $\Omega$, by a finite covering technique we conclude that $u(x, t)$ is Hölder continuous in $Q$ as long as $\operatorname{dist}\left\{Q, S_{T}\right\}>0$. This completes our proof for Theorem 2.1.

Using the second part of Lemma 3.2 and the same argument as above, we can establish Theorem 2.2.

\section{Applications.}

In this Section, we shall use the results obtained in the preceding section to show global existence of a classical solution for nonlinear parabolic Volterra integrodifferential equations. Consider the following problem:

$$
u_{t}=\frac{\partial}{\partial x_{i}}\left[a_{i j}(x, t, u) \frac{\partial u}{\partial x_{j}}\right]+\int_{0}^{t} \frac{\partial}{\partial x_{i}}\left[b_{i j}(x, t, \tau, u) \frac{\partial u}{\partial x_{j}}\right] d \tau \quad \text { in } Q_{T},
$$

$$
u(x, t)=g(x, t) \quad \text { on } S_{T}
$$

$$
u(x, 0)=u_{0}(x) \quad \text { on } \Omega .
$$

Theorem 4.1. Assume that the coefficients $a_{i j}(x, t, s)$ satisfy the ellipticity condition (2.3), $a_{i j}, \frac{\partial}{\partial x_{k}}\left(a_{i j}\right), \frac{\partial}{\partial u}\left(a_{i j}\right) \in C^{\alpha, \frac{\alpha}{2}, \beta}\left(\bar{Q}_{T} \times R^{1}\right)$, and $b_{i j}, \frac{\partial}{\partial x_{k}}\left(b_{i j}\right)$,

$\frac{\partial}{\partial u}\left(b_{i j}\right) \in C^{\alpha, \frac{\alpha}{2}, \frac{\alpha}{2}, \beta}\left(\bar{Q}_{T} \times[0, T] \times R^{1}\right)$, where $\alpha, \beta \in(0,1)$. Moreover, there exists a function $\psi(x, t) \in C^{2+\gamma, 1+\frac{\gamma}{2}}\left(\bar{Q}_{T}\right)(\gamma=\alpha \beta)$ such that $\psi(x, t)=g(x, t)$ 
on $S_{T}$ and $\psi(x, 0)=u_{0}(x)$ on $\Omega$. Then the problem (4.1)-(4.3) exists a unique classical solution.

Proof. Local existence of a classical solution is standard from the theory of parabolic equations and contraction mapping principle. By using the method of continuity, we know that global existence relies on an a priori estimate in the space $C^{2+\alpha, 1+\frac{\alpha}{2}}\left(\bar{Q}_{T}\right)$. Multiplying the Equation (4.1) by $u-g(x, t)$, we can easily obtain

$$
\|u\|_{L^{2}\left(0, T ; H^{1}(\Omega)\right)} \leq C .
$$

From Theorem 2.2, we know

$$
\|u\|_{C^{\alpha, \frac{\alpha}{2}}\left(\bar{Q}_{T}\right)} \leq C .
$$

If we rewrite the Equation (4.1) in nondivergence form, then the coefficients are Hölder continuous. Moreover, their Hölder norms are bounded by known data. Using the global Schauder estimate for parabolic Volterra integrodifferential equations (cf. [16]), we obtain

$$
\|u\|_{C^{2+\gamma, 1+\frac{\gamma}{2}\left(\bar{Q}_{T}\right)}} \leq C \quad(\gamma=\alpha \beta)
$$

where $C$ depends only on known data. Consequently, we have the desired result.

Acknowledgment. The authors thank Professor G. Lieberman for his helpful comments.

\section{References}

[1] G. Andrews, On existence of solutions to the equation $u_{t t}=u_{x x t}+\sigma\left(u_{x}\right)_{x}$, J. Diff. Eqs., 35 (1980), 200-231.

[2] D.S. Cohen and A.B. White, Sharp fronts due to diffusion and viscoelastic relaxation in polymers, SIAM J. Appl. Math., 51 (1991), 472-485.

[3] S. Campanato, Equazioni paraboliche del secondo ordine e spazi $\mathcal{L}^{2, \theta}(\Omega, \delta)$, Annali di Matem. Pura e Appl., 73 (1966), 55-102.

[4] M.G. Crandall, S.O. Londen and J.A. Nohel, An abstract nonlinear Volterra integrodifferential equation, J. Math. Anal. Appl., 64 (1978), 701-735.

[5] C.M. Dafermos, The mixed initial boundary value problem for the equations of nonlinear one dimensional visco-elasticity, J. Diff. Eqs., 6 (1969), 71-86.

[6] G. Da Prato and M. Iannelli, Existence and regularity for a class of integrodifferential equations of parabolic type, J. Math. Anal. Appl., 112 (1985), 36-55. 
[7] A. Friedman, Mathematics in Industrial Problems, Springer-Verlag, New York, 1992.

[8] M. Giaquinta, Multiple Integrals in the Calculus of Variations and Nonlinear Elliptic Systems, Princeton University Press, New Jersey, 1983.

[9] J. Moser, On a pointwise estimate for parabolic differential equations, Comm. on Pure and Applied Mathematics, 24 (1971), 727-740.

[10] J.A. Nohel, Nonlinear Volterra equations for heat flow in materials with memory, Integral and functional differential equations, Lecture notes in pure and applied mathematics, edited by T.L. Herdman, S.M. Rankin, III, H.W. Stech, Marcel Dekker Inc., 1981.

[11] G.M. Troianiello, Elliptic Differential Equations and Obstacle Problems, Plenum Press, New York, 1987.

[12] O.A. Ladyzenskaja, V.A. Solonnikov and N.N. Ural'ceva, Linear and Quasilinear Equations of Parabolic type, AMS translation Monograph, Vol. 23, Providence, Rhode Island, 1968.

[13] M. Renardy, W.J. Hrusa and J.A. Nohel, Mathematical Problems in Viscoelasticity, Longman Scientific and Technical, New York, 1987.

[14] M. Struwe, On the Hölder continuity of bounded weak solutions of quasilinear parabolic systems, Manscripta Mathematica, 35 (1981), 125-145.

[15] H.M. Yin, The classical solutions for nonlinear integrodifferential equations, J. Integral Equations and Applications, 1 (1988), 249-263.

[16] - On parabolic Volterra equations in several space dimensions, SIAM J. on Mathematical Analysis, 22 (1991), 1723-1737.

[17] Weak and classical solutions of some Volterra integrodifferential equations, Communication in P.D.E., 17 (1992), 1369-1385.

[18], $\mathcal{L}^{2, \mu}$ theory for parabolic equations and applications, J. of Partial Differential Equations, 10(1) (1997).

Received June 15, 1995 and revised November 10, 1995. The work of the first author is partially supported by NSF grant DMS 92-24935.

UNIVERSITY OF NotRE DAME

Notre DAME, IN 46556

E-mail address: Bei.Hu.1@nd.edu or Hong-Ming.Yin.3@nd.edu 